\title{
Licence to kill senescent cells in idiopathic pulmonary fibrosis?
}

\author{
Arnaud A. Mailleux ${ }^{1,2}$ and Bruno Crestani ${ }^{1,2,3}$
}

Affiliations: ${ }^{1}$ Inserm UMR1152, Medical School Xavier Bichat, Paris, France. ${ }^{2}$ Université Paris Diderot, Sorbonne Paris Cité, Département Hospitalo-Universitaire FIRE (Fibrosis, Inflammation and Remodeling) and LabEx Inflamex, Paris, France. ${ }^{3}$ APHP, Hôpital Bichat, Service de Pneumologie A, Rare Pulmonary Disease Reference Center, Paris, France.

Correspondence: Bruno Crestani, Service de Pneumologie A, Hôpital Bichat, 46 rue Henri Huchard, 75877 Paris cedex 18, France. E-mail: bruno.crestanidaphp.fr

@ERSpublications

Targeting senescent cells may prove useful in the future treatment of idiopathic pulmonary fibrosis http://ow.ly/rHpn30dvsuP

Cite this article as: Mailleux AA, Crestani B. Licence to kill senescent cells in idiopathic pulmonary fibrosis? Eur Respir J 2017; 50: 1701360 [https://doi.org/10.1183/13993003.01360-2017].

Idiopathic pulmonary fibrosis (IPF) is a rare chronic fibrotic pulmonary disease of unknown aetiology, which results in the progressive destruction of lung with a very poor median survival of 3 years after diagnosis. According to the current paradigm, IPF results from progressive alterations of alveolar epithelial cells leading to the recruitment of mesenchymal cells to the alveolar regions of the lung with secondary deposition of extracellular matrix, and destruction of the normal lung structure and physiology [1]. The anarchic epithelial repair observed in IPF is also accompanied by reactivation of signalling pathways involved in fetal lung development [2]. IPF develops in a genetically susceptible individual, and is promoted by interaction with environmental agents such as inhaled particles, tobacco smoke, inhaled pollutants, and viral and bacterial agents. Two drugs are currently available for the treatment of pulmonary fibrosis: pirfenidone, a small molecule that probably targets lung fibroblasts through multiple mechanisms; and nintedanib, a multitarget tyrosine kinase inhibitor [1].

IPF has been clearly associated with ageing. Ageing is both a susceptibility marker and a major driver of the disease, through mechanisms that have not yet been fully elucidated. Cellular senescence is one of the nine hallmarks of ageing, besides genomic instability, telomere attrition, epigenetic alterations, loss of proteostasis, deregulated nutrient sensing, mitochondrial dysfunction, altered intercellular communication and stem cell exhaustion [3,4]. Cellular senescence was first described half a century ago as "the limited in vitro lifetime of human diploid cell strains" in Hayflick and Moorhead's seminal works [5, 6]. Cellular senescence is characterised by a supposedly irreversible cell cycle arrest (even in the presence of mitogenic signals). This cellular state is associated with apoptosis resistance, and the secretion of many cytokines, growth factors and proteases: the so-called senescence-associated secretory phenotype (SASP) [7], which may impact the neighbouring cells and tissue microenvironment. The composition of the SASP depends on the cell type of origin as well as the senescence-inducing stressor [8]. Many triggers of cellular senescence have been identified. Cellular senescence was first associated with telomere attrition leading to a persistent DNA damage response [9]. Additional DNA damage response-independent stressors associated with ageing, such as oxidative stress and proteostasis dysregulation, can also trigger cellular senescence $[3,9]$. Identification of a senescent cell in a tissue is a difficult issue. Canonical markers of senescence include senescence-associated $\beta$-galactosidase $(\mathrm{SA} \beta \mathrm{G})$ activity, which reflects the accumulation of endogenous lysosomal $\beta$-galactosidase $[10,11]$ as well as the induction of cell cycle-related inhibitory

Received: July 062017 | Accepted: July 072017

Conflict of interest: Disclosures can be found alongside this article at erj.ersjournals.com

Copyright @ERS 2017 
proteins such as P53, P21 and P16 ${ }^{\mathrm{INK} 4 a}$ in senescent cells [9]. In addition to DNA damage response markers, senescent cells may display heterochromatin foci that contribute to silencing of proliferation-promoting genes in order to epigenetically lock down the cell in this prolonged nonproliferative state $[12,13]$. No single marker may define a cell as senescent since none of these markers are exclusive to cellular senescence; only the association of those markers should be used to evaluate a senescent cell [9].

Cellular senescence has important physiological roles. Recent studies have shown that cellular senescence is involved during the ontogenesis of the limb, kidney and inner ear $[14,15]$. Senescence is also needed for normal wound healing. Demaria et al. [16] showed that senescent fibroblasts and endothelial cells appear very early in response to a cutaneous wound, where they accelerate wound closure by inducing myofibroblast differentiation after a cutaneous wound and limit skin fibrosis. Similarly, JUN and LAU [17] showed that senescent fibroblasts accumulate in granulation tissues of healing cutaneous wounds and express antifibrotic genes. The secretory repertoire of senescent cells promotes the growth of the surrounding cells involved in microenvironment remodelling during organ morphogenesis or wound healing [14-16], and then attracts phagocytic immune cells to trigger the removal of the senescent cell and promote tissue regeneration [9]. Senescence is also a crucial barrier against cancer development since this process potentially disables cells accumulating severe cellular damage and potentially deleterious mutations [9]. These data suggest that cellular senescence is an example of antagonistic pleiotropy in which natural selection favours processes that are beneficial early in life, even if they cause harmful effects later in post-reproduction life [1-3]. Cellular senescence would act beneficially early in life during tissue morphogenesis and during normal wound healing upon tissue damage, but detrimentally later in aged organisms by causing aberrant tissue remodelling as a consequence of persistent SASP [9]. Similarly, senescence would be beneficial when it is transient and efficiently resolved but pathological when chronic and unresolved.

Accumulation of senescent cells has been associated with structural changes associated with ageing. Recent data, including the article by LeHMANN et al. [18] published in this issue of the European Respiratory Journal, have linked cellular senescence with lung fibrosis development [19]. Indeed, IPF is characterised by increased epithelial [20-22] and mesenchymal/fibroblast [19] senescence. LeHMANN et al. [18] focused on epithelial senescence in IPF, confirming that lung epithelial cells display senescence markers such as SABG, P21, P16 ${ }^{\mathrm{INK} 4 \mathrm{a}}$ and DNA damage response markers in IPF patients. Interestingly, they also showed that the expression of senescence markers involved both alveolar epithelial type II cells (AEC2), and cytokeratin-5- and -7-positive basal cells in bronchiolised IPF distal airways, and that primary AEC2 derived from IPF lung also retained senescence markers in vitro. Similarly, they confirmed that senescence features are detected in experimental models of lung fibrosis upon bleomycin treatment in mice with an increase in senescence markers and secretion of SASP factors in primary AE2C derived from mouse fibrotic lungs $[19,23,24]$. Other investigators observed that IPF fibroblasts in culture retain markers of senescence [25]. Interestingly, many senescence triggers, such as telomere attrition, oxidative stress and proteostasis dysregulation, have been reported in sporadic and familial forms of IPF [26, 27].

Cellular senescence is being increasingly recognised as a therapeutic target in many diseases, including IPF. Even though senescent cells are resistant to apoptosis, these cells express high levels of both pro- and antiapoptotic factors, meaning that these cells are in fact on the verge of undergoing apoptosis. There is the possibility to induce their apoptosis through the inhibition of antiapoptotic factors [8]. Drugs that can selectively induce the death of senescent cells are called "senolytic". In the current study, LeHMANN et al. [18] took advantage of a recently developed senolytic drug combination, dasatinib and quercetin (D+Q). Dasatinib is a tyrosine kinase inhibitor, while quercetin is a natural flavonol that targets phosphoinositide 3-kinase, among other kinases [8]. They showed that the D+Q senolytic combination depleted senescent cells by inducing apoptosis and reduced SASP factors in mouse primary AEC2 derived from bleomycin-treated fibrotic lungs. These results were also confirmed in the ex vivo model of precision-cut lung slices obtained from bleomycin-treated fibrotic mouse lungs [18]. Another recent study by SCHAFER et al. [19] undertook a similar approach by administering the D+Q cocktail in vivo during the fibrotic phase in bleomycin-treated animals. This senolytic combination did decrease fibrosis burden in these animals [19]. The beneficial effect of eliminating senescent cells during lung fibrosis was also confirmed in vivo by using an elegant genetic approach to specifically induce apoptosis in $p 16^{\text {ink } 4 a}$-positive/senescent cells in mice treated with bleomycin [19]. A recent study also showed that mitigating P53 localisation and function in senescent cells could specifically trigger their elimination by apoptosis and restore tissue homeostasis in mice treated with chemotherapy agents and in aged animals [28].

Together, the studies by Lehmann et al. [18] and SCHAFER et al. [19] strongly suggest that cellular senescence is a salient feature of lung fibrosis, and that targeting/elimination of these cells could be beneficial. These observations raise many issues. From a biological perspective, it will be important to 
decipher the mechanisms leading to the accumulation of senescent epithelial cells in IPF. Specifically, the potential contribution of immunosenescence with a secondary impairment of the processes leading to removal of senescent cells [29] should be further studied. From a clinical perspective, the efficiency of senescent cell removal regarding fibrogenesis should be further validated in other experimental models of lung fibrosis before moving to patients. Indeed, we still cannot exclude that senolytic drugs could be detrimental in IPF patients, for various reasons. Firstly, senolytic drugs may target senescent fibroblasts, which have been shown to have fibrosis-limiting effects in the skin, heart and liver [9, 16, 30]; interestingly, senescence is mainly associated with the mesenchymal lineage (myofibroblasts) in these tissues $[9,16,30]$. Similarly, increased senescence of fibroblasts may decrease experimental lung fibrosis in mice [31, 32]. However, as suggested by LeHMANN et al. [18], senescence seems to predominate in epithelial cells in IPF; therefore, elimination of senescent antifibrotic fibroblasts should not be a limiting factor. Secondly, dasatinib was recently shown to induce pulmonary endothelial damage, with an increased susceptibility to pulmonary hypertension in rodent experimental models and in patients with chronic myeloid leukaemia treated with dasatinib [33]. Since pulmonary hypertension is a well-recognised comorbidity in IPF [34], the use of dasatinib in IPF patients could have potential aggravating effects in the long term. Finally, and this is probably more worrying, senolytic drug treatment may result in massive epithelial cell depletion by apoptosis, which could trigger diffuse alveolar damage and acute exacerbation, since the regenerative capacity of epithelial cells in IPF patients is impaired $[1,35]$. A way to investigate and circumvent those harmful caveats in IPF would be to study the effect of senolytic drugs on precision-cut IPF lung slices in vitro. Such an approach has been already validated to test small molecules in chronic obstructive pulmonary disease-derived lung slices [36].

In conclusion, we should be very cautious before translating the beneficial effects of senolytic drugs from mice to men. Senolytic drugs could be a promising adjuvant therapy in IPF. However, the challenge will be to confirm in clinical trials their true beneficial effects in IPF since senolytic drugs could be potentially harmful in these fragile patients.

\section{References}

$1 \quad$ Richeldi L, Collard HR, Jones MG. Idiopathic pulmonary fibrosis. Lancet 2017; 389: 1941-1952.

2 Selman M, Lopez-Otin C, Pardo A. Age-driven developmental drift in the pathogenesis of idiopathic pulmonary fibrosis. Eur Respir J 2016; 48: 538-552.

3 Meiners S, Eickelberg O, Konigshoff M. Hallmarks of the ageing lung. Eur Respir J 2015; 45: 807-827. Lopez-Otin C, Blasco MA, Partridge L, et al. The hallmarks of aging. Cell 2013; 153: 1194-1217.

Hayflick L. The limited in vitro lifetime of human diploid cell strains. Exp Cell Res 1965; 37: 614-636.

Hayflick L, Moorhead PS. The serial cultivation of human diploid cell strains. Exp Cell Res 1961; 25: 585-621.

Chilosi M, Carloni A, Rossi A, et al. Premature lung aging and cellular senescence in the pathogenesis of idiopathic pulmonary fibrosis and COPD/emphysema. Transl Res 2013; 162: 156-173.

8 Kirkland JL, Tchkonia T. Cellular senescence: a translational perspective. EBioMedicine 2017 [in press https://doi. org/10.1016/j.ebiom.2017.04.013].

9 Munoz-Espin D, Serrano M. Cellular senescence: from physiology to pathology. Nat Rev Mol Cell Biol 2014; 15: 482-496.

10 Young AR, Narita M, Ferreira M, et al. Autophagy mediates the mitotic senescence transition. Genes Dev 2009; 23: 798-803.

11 Lee BY, Han JA, Im JS, et al. Senescence-associated $\beta$-galactosidase is lysosomal $\beta$-galactosidase. Aging Cell 2006; 5: $187-195$.

12 Kosar M, Bartkova J, Hubackova S, et al. Senescence-associated heterochromatin foci are dispensable for cellular senescence, occur in a cell type- and insult-dependent manner and follow expression of p16 ${ }^{\text {ink4a }}$. Cell Cycle 2011; 10: $457-468$.

13 Narita M, Narita M, Krizhanovsky V, et al. A novel role for high-mobility group A proteins in cellular senescence and heterochromatin formation. Cell 2006; 126: 503-514.

14 Munoz-Espin D, Canamero M, Maraver A, et al. Programmed cell senescence during mammalian embryonic development. Cell 2013; 155: 1104-1118.

15 Storer M, Mas A, Robert-Moreno A, et al. Senescence is a developmental mechanism that contributes to embryonic growth and patterning. Cell 2013; 155: 1119-1130.

16 Demaria M, Ohtani N, Youssef SA, et al. An essential role for senescent cells in optimal wound healing through secretion of PDGF-AA. Dev Cell 2014; 31: 722-733.

17 Jun JI, Lau LF. The matricellular protein CCN1 induces fibroblast senescence and restricts fibrosis in cutaneous wound healing. Nat Cell Biol 2010; 12: 676-685.

18 Lehmann M, Korfei M, Mutze K, et al. Senolytic drugs target alveolar epithelial cell function and attenuate experimental lung fibrosis ex vivo. Eur Respir J 2017; 50: 1602367.

19 Schafer MJ, White TA, Iijima K, et al. Cellular senescence mediates fibrotic pulmonary disease. Nat Commun 2017; 8: 14532 .

20 Disayabutr S, Kim EK, Cha SI, et al. miR-34 miRNAs regulate cellular senescence in type II alveolar epithelial cells of patients with idiopathic pulmonary fibrosis. PLoS One 2016; 11: e0158367.

21 Minagawa S, Araya J, Numata T, et al. Accelerated epithelial cell senescence in IPF and the inhibitory role of SIRT6 in TGF-beta-induced senescence of human bronchial epithelial cells. Am J Physiol Lung Cell Mol Physiol 2011; 300: L391-L401.

22 Kuwano K, Kunitake R, Kawasaki M, et al. P21Waf1/Cip1/Sdi1 and p53 expression in association with DNA strand breaks in idiopathic pulmonary fibrosis. Am J Respir Crit Care Med 1996; 154: 477-483. 
23 Aoshiba K, Tsuji T, Kameyama S, et al. Senescence-associated secretory phenotype in a mouse model of bleomycin-induced lung injury. Exp Toxicol Pathol 2013; 65: 1053-1062.

$24 \mathrm{Xu}$ J, Gonzalez ET, Iyer SS, et al. Use of senescence-accelerated mouse model in bleomycin-induced lung injury suggests that bone marrow-derived cells can alter the outcome of lung injury in aged mice. J Gerontol A Biol Sci Med Sci 2009; 64: 731-739.

25 Yanai H, Shteinberg A, Porat Z, et al. Cellular senescence-like features of lung fibroblasts derived from idiopathic pulmonary fibrosis patients. Aging (Albany NY) 2015; 7: 664-672.

26 Kannengiesser C, Borie R, Menard C, et al. Heterozygous RTEL1 mutations are associated with familial pulmonary fibrosis. Eur Respir J 2015; 46: 474-485.

27 Borie R, Crestani B. Prevalence of telomere shortening in familial and sporadic pulmonary fibrosis is increased in men. Am J Respir Crit Care Med 2009; 179: 1073.

28 Baar MP, Brandt RM, Putavet DA, et al. Targeted apoptosis of senescent cells restores tissue homeostasis in response to chemotoxicity and aging. Cell 2017; 169: 132-147.

29 Murray MA, Chotirmall SH. The Impact of immunosenescence on pulmonary disease. Mediators Inflamm 2015; 692546.

30 Krizhanovsky V, Yon M, Dickins RA, et al. Senescence of activated stellate cells limits liver fibrosis. Cell 2008; 134: $657-667$.

31 Li Y, Liang J, Yang T, et al. Hyaluronan synthase 2 regulates fibroblast senescence in pulmonary fibrosis. Matrix Biol 2016; 55: 35-48.

32 Cui H, Ge J, Xie N, et al. miR-34a inhibits lung fibrosis by inducing lung fibroblast senescence. Am J Respir Cell Mol Biol 2017; 56: 168-178.

33 Guignabert C, Phan C, Seferian A, et al. Dasatinib induces lung vascular toxicity and predisposes to pulmonary hypertension. J Clin Invest 2016; 126: 3207-3218.

34 Harari S, Elia D, Humbert M. Pulmonary hypertension in parenchymal lung diseases: any future for new therapies? Chest 2017 [in press https://doi.org/10.1016/j.chest.2017.06.008].

35 MacKenzie B, Korfei M, Henneke I, et al. Increased FGF1-FGFRc expression in idiopathic pulmonary fibrosis. Respir Res 2015; 16: 83.

36 Uhl FE, Vierkotten S, Wagner DE, et al. Preclinical validation and imaging of Wnt-induced repair in human 3D lung tissue cultures. Eur Respir J 2015; 46: 1150-1166. 